\title{
Accès à l'eau et clientélisme dans le Nordeste brésilien
}

\author{
Anne-Laure Collard ${ }^{1}$, Julien Burte ${ }^{2}$, Florence Pinton ${ }^{3}$, Pedro Roberto Jacobi ${ }^{4}$, Jean-Yves Jamin ${ }^{5}$ \\ 1 Doctorante en sociologie, AgroParisTech, UFR Sociologie, 75005 Paris, France \\ 2 Hydrologue, UFC, PPGEA, Fortaleza, Brésil \\ 3 Sociologue, AgroParisTech, UFR Sociologie, 75005 Paris, France \\ 4 Sociologue, PROCAM-USP, São Paulo, Brésil \\ 5 Agronome, CIRAD, UMR G-Eau, 34398 Montpellier, France
}

\begin{abstract}
La question de l'accès à l'eau est un enjeu central des politiques de développement dans les pays du Sud et plus encore, dans les pays soumis à des régimes hydriques capricieux. À côté de grands projets hydrauliques, on voit émerger des efforts de la part de la puissance publique pour favoriser la mise en place de systèmes techniques locaux dans lesquels sont impliquées les populations. C'est le cas du Nordeste, région semi-aride du Brésil, où l'accès à l'eau est monopolisé par les oligarchies agraires. Par une étude de terrain comparant trois communautés confrontées à des aménagements dans le domaine de l'accès à l'eau domestique, les auteurs de l'article montrent comment le transfert de technologie n'arrive pas à se dégager de rapports de clientélisme hérités de l'histoire. NSS a déjà publié sur l'action collective et la gestion de l'eau (NSS, 17, 3, 248-256 ; NSS, 20, 3, 286-296), cette nouvelle étude de cas met en lumière ce qui se joue dans les rapports entre l'État, les acteurs locaux et les membres de ces communautés.
\end{abstract}

La Rédaction

\section{Mots-clés :}

action publique ;

clientélisme ; gestion de l'eau ; objet sociotechnique ; semi-aride

\section{Keywords:}

clientelism; water management; socio-technical object; semi-arid environment
Résumé - Dans le Nordeste brésilien semi-aride, les récents projets d'approvisionnement en eau des petites communautés rurales affichent une rupture avec le modèle traditionnel d'action publique basé sur la grande hydraulique. De la lutte contre la sécheresse, on est passé à la lutte contre la pauvreté, et l'intervention se veut désormais localisée, décentralisée et participative. La question est de savoir si le nouvel accès à l'eau domestique modifie les relations de dépendance locales. L'étude du « réseau d'eau domestique » en tant qu'objet sociotechnique permet de se poser la question de son appropriation par les populations locales et les potentats locaux. L'analyse des récits de vie montre que, malgré des avancées, le clientélisme reste au cœur des dynamiques locales, même si sa nature évolue.

Abstract - Access to water and clientelism in the semi-arid Brazilian Northeast. In the semi-arid Brazilian Northeast, the recent water supply projects in small rural communities break away from the traditional model of public action based on - large-scale hydraulics -. Emphasis in these projects has shifted from the fight against drought to the fight against poverty and interventions now aim to be localized, decentralized and participatory. However, does this new access to domestic water modify local dependency relations? Studying the "domestic water network" as a socio-technical system questions its actual appropriation by local populations. The analysis of life narratives shows that despite improvements for the local population, these projects are still rooted in a clientelistic heritage and topdown logic. Clientelism remains at the heart of local dynamics even though its nature is progressively changing.

\section{Introduction : l'eau comme instrument de pouvoir historique dans le Sertão}

De nombreux auteurs ont développé une posture critique vis-à-vis des politiques publiques de développement de la région semi-aride du Brésil (De Castro, 1946 ;
Andrade, 1986 ; Durousset, 2001 ; Abramovay, 2002). Comme d'autres pays confrontés à la rareté de l'eau, tels que l'Inde, le Sénégal, le Maroc ou l'Algérie (Marzouk, 1991 ; Kuper et al., 2009 ; Kuper, 2011 ; Jamin et al., 2011), le Nordeste du Brésil n'a pas échappé aux solutions de grande hydraulique, tant pour sécuriser l'alimentation

Auteur correspondant : A.-L. Collard, collard.annelaure@gmail.com 
en eau potable des populations que pour développer l'irrigation. Le discours officiel justifie ces solutions par la contrainte forte des aléas climatiques.

En effet, plus de $80 \%$ du Nordeste est semi-aride et marqué par l'irrégularité spatiale et temporelle des précipitations (Mainguet, 2003). L'approvisionnement en eau des populations rurales dépend essentiellement de petites ressources, superficielles ou souterraines (nappes alluviales, aquifère du socle).

Historiquement, les fazendeiros (grands propriétaires fonciers) bénéficient d'un accès privilégié aux ressources en eau, puisque la division foncière a été réalisée perpendiculairement aux cours d'eau (Molle, 1994) et que les héritages perpétuent ce schéma. Les ouvrages hydrauliques renforcent cette logique. Ils représentent un véritable instrument de domination et de pouvoir, $\mathrm{d}^{\prime}$ une part parce qu'ils sont traditionnellement détenus par les fazendeiros qui les ont fait construire sur leurs terres pour se les approprier, d'autre part parce que la politique publique de l'eau suit une logique descendante (topdown). Cela induit des effets d'exclusion et de marginalisation des populations (Cohen et Duqué, 1989). Dans les vallées, l'exploitation des petits aquifères alluviaux peu profonds, via des cacimbões (puits en maçonnerie) ou des cacimbas (excavations dans le lit de la rivière) est restreinte, pour l'essentiel, aux propriétaires riverains. Les seuls accès publics à l'eau sont les rares points où les chemins publics traversent les rivières. Lors des périodes de sécheresse, l'approvisionnement en eau potable de la population dépend soit des élus locaux qui distribuent l'eau par des camions-citernes spécialement affrétés, soit des « faveurs » ${ }^{1}$ (Geffray, 1996) des grands propriétaires qui autorisent ou pas l'accès à la ressource sur leurs terres, selon une règle locale ${ }^{2}$. De plus, la population reste enfermée dans la pauvreté car la mauvaise répartition de l'eau ne permet ni un abreuvement ni une production fourragère suffisants pour maintenir productifs les troupeaux, principal capital économique des ruraux. Les périodes de sécheresse sont donc propices à la reproduction de rapports de domination.

À l'intérieur du Nordeste, dans le Sertão, les facteurs structurels comme le clientélisme (De Castro, 1946; Amman, 1985) ou l'inégalité foncière (Durousset et Duqué, 1998) sont mis en avant pour rendre compte de la pauvreté et de l'inefficacité des politiques de l'eau.

Depuis le début des années 1990, le modèle d'action publique se réoriente vers des projets de développement

\footnotetext{
1 Geffray (1996) analyse les valeurs symboliques et le cadre imaginaire du clientélisme, au-delà du cadre marchand. Les dominés perçoivent les gestes des dominants comme des faveurs, des présents, et pas comme un «simple dû ».

2 Il n'existe pas de droits d'eau formalisés. Mais, le propriétaire de la terre est communément considéré comme « dono da água»-propriétaire de l'eau courante ou stockée.
}

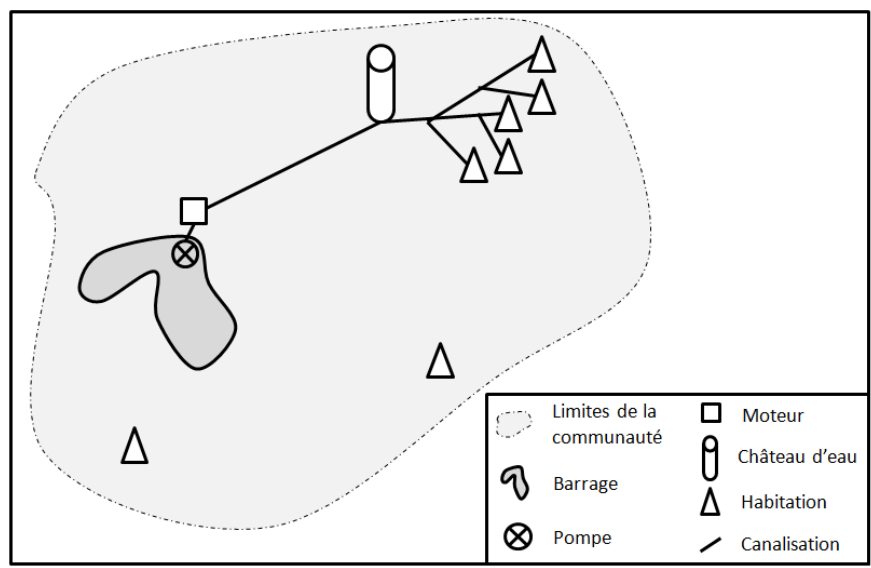

Fig. 1. Schéma type d'un réseau d'adduction d'eau.

Le barrage est public. Il est construit sur les terres de la communauté. Les habitations trop distantes du château d'eau ne sont pas reliées au réseau.

local : forages dans l'aquifère de socle, petites retenues collinaires appelées açudes et, surtout, réseaux d'eau potable. L'objectif est de sécuriser l'accès à l'eau des petits villages pour réduire le recours aux camions-citernes qui, en plus d'être très coûteux pour les finances publiques (Araujo et al., 2005), est un symbole de la dépendance des populations et de leur domination par les potentats locaux (Duqué, 2008).

Les réseaux d'adduction d'eau potable sont constitués d'un système de pompage électrique à partir d'un barrage ou d'une nappe, d'un château d'eau et d'un réseau de canalisations qui dessert les habitations (Fig. 1). Les habitats les plus isolés ne sont pas reliés, l'installation de réseaux étendus serait trop coûteuse. En réalité, dans le cas de notre étude, l'eau du réseau n'est pas vraiment potable car il n'y a ni suivi ni traitement de l'eau. On privilégiera donc ici l'expression « eau domestique », même si l'eau du réseau est consommée par les habitants.

Les projets d'adduction d'eau portent le nom de « projet São José » dans l'État du Ceará. Ils sont financés grâce à un emprunt du Brésil auprès de la Banque mondiale (Soares, 1997) et à une participation des populations locales $(10 \%)$. Celle-ci se traduit par un apport financier ou la fourniture de main-d'œuvre - option souvent choisie - pour la construction du réseau ; elle doit en garantir l'appropriation par les paysans.

Ces politiques de construction de petites infrastructures locales atteignent-elles l'objectif affiché de diminuer la dépendance des populations rurales pour leur approvisionnement en eau ? Le propos de cet article ${ }^{3}$ est ainsi

3 Cet article s'inscrit dans le cadre d'une thèse intitulée «Réappropriation des usages et de l'accès à l'eau dans le Nordeste brésilien » et soutenue par la Funceme et le Cirad. Le travail porte sur les dynamiques locales liées à l'introduction de réseau d'adduction d'eau dans trois communautés rurales. 
de poser la question du rôle des objets techniques, introduits par les politiques publiques, dans la réduction des rapports clientélistes.

\section{Le réseau d'adduction d'eau domestique comme objet sociotechnique}

La posture de ce travail est de considérer que les rapports sociaux vécus localement s'insèrent dans des dynamiques dites "englobantes", afin de dépasser l'approche locale de la communauté et de saisir sa spécificité par référence à des logiques qui lui sont extérieures (Pinton, 2009). Notre hypothèse est que l'introduction d'un système technique, même simple, modifie le comportement et la vie sociale des individus. En effet, ces systèmes techniques sont des objets hybrides, puisqu'ils sont aussi des constructions sociales, soumises à des formes d'irrationalité, de croyance et de discours, quelle que soit la modernité des sociétés concernées (Latour, 1991). C'est pourquoi nous cherchons à comprendre les logiques d'action des acteurs locaux à partir de leurs représentations. Ces dernières sont comprises comme un mode de connaissance cohérent (Garfinkel, 1967), qui s'attache à un système de valeurs, de notions et de pratiques (Moscovici, 1961).

Le « réseau d'eau » est considéré comme un objet à travers et par lequel les acteurs entrent en interaction (Vinck, 1999). On le définit comme objet sociotechnique, ce qui permet de s'intéresser aux médiations et à la nature des échanges entre entités humaines (Akrich, 1989) ou non humaines (Latour, 1991).

Ce cadre théorique s'articule avec les enjeux de gestion concertée de l'eau qui, pour sa réussite, demande aux différents acteurs - politiques, experts, société civile coresponsables de celle-ci, un effort d'apprentissage collectif (Harmonicop, 2005 ; Jacobi, et al., 2009). La construction d'un espace de dialogue, autour de la gestion d'un réseau d'eau, soulève des questions cognitives, car l'enjeu n'est pas seulement l'acquisition de connaissances, mais aussi la compréhension des différentes perceptions de cette même situation (Harmonicop, 2005), à travers la construction de nouvelles relations entre acteurs.

Comme le suggèrent Venot et Cecchi (2011) lorsqu'ils analysent les contradictions entre l'action publique et les attentes locales autour des usages des petits réservoirs en Afrique subsaharienne, considérer les objets d'aménagement du territoire comme des vecteurs de changement social, selon une approche pluridisciplinaire, permet d'analyser les opportunités que ces derniers offrent aux populations locales. Notre équipe de sociologues, agronome et hydrologue s'inscrit dans cette perspective pluridisciplinaire qui permet d'aller au-delà de l'analyse de la stricte dimension technique d'un projet, pour suivre son immersion sociale: en mettant l'accent sur les logiques d'action des acteurs du réseau sociotechnique, elle ouvre de nouvelles perspectives pour analyser les impacts d'un modèle d'action publique.

Les réseaux d'eau domestique sont des objets d'analyse intéressants car ils concernent l'ensemble de la population, contrairement aux réseaux d'irrigation qui ne s'adressent ici qu'à une partie des agriculteurs. De plus, leur installation et leur pérennité nécessitent une gestion collective ou des décisions collectives sur cette gestion. Dans le cas étudié, les réseaux d'acteurs s'inscrivent dans un contexte sociohistorique particulier qui s'articule autour de trois grandes catégories d'acteurs : l'oligarchie agraire, composée des élus locaux et des grands propriétaires fonciers qui entretiennent une relation de domination avec les populations locales ; les porteurs $\mathrm{du}$ modèle d'action publique - élus politiques et techniciens d'État - qui veulent garantir l'approvisionnement en eau des populations; et enfin, les associations communautaires, entités formalisées regroupant les habitants, qui portent les demandes de projet.

Au-delà de l'intérêt de mobiliser le concept d'objet sociotechnique, que d'autres ont utilisé, l'originalité de ce travail se situe dans le dispositif de comparaison de situations. Nous avons retenu un échantillon de trois communautés (Fig. 2) : Cachoeira do Germano (CG), Lagoa São Miguel (LSM) et Quinim, qui appartiennent au même municipe ${ }^{4}$, celui de Quixeramobim.

La communauté est choisie comme échelle d'analyse, car elle constitue au Brésil le niveau territorial auquel sont mis en œuvre les projets, à travers les associations. Le terme "communauté » vient des valeurs de la théologie de la libération - fraternité, spiritualité-, que l'Église défend à travers les initiatives locales (Bleil, 2003 ; Tonneau et al., 2010). Après la fin de la dictature, le gouvernement retient la communauté comme entité sociale pertinente pour accueillir les actions des politiques publiques. La communauté n'a pas de statut juridique, le plus petit maillon administratif étant le municipe. C'est pourquoi, pour pouvoir bénéficier de projets, les populations rurales doivent s'organiser en association communautaire.

À partir d'études de cas dans l'État du Ceará, situé dans le Nordeste brésilien, nous analyserons les transformations induites par les réseaux d'eau domestique sur les relations de dépendance des communautés pour leur approvisionnement en eau, et sur les opportunités qu'elles leur offrent de modifier leurs rapports avec les pouvoirs locaux. Après avoir présenté la méthodologie

\footnotetext{
4 Le municipe est l'équivalent d'une commune en France. Sa superficie peut atteindre celle d'un canton ou d'un département.
} 


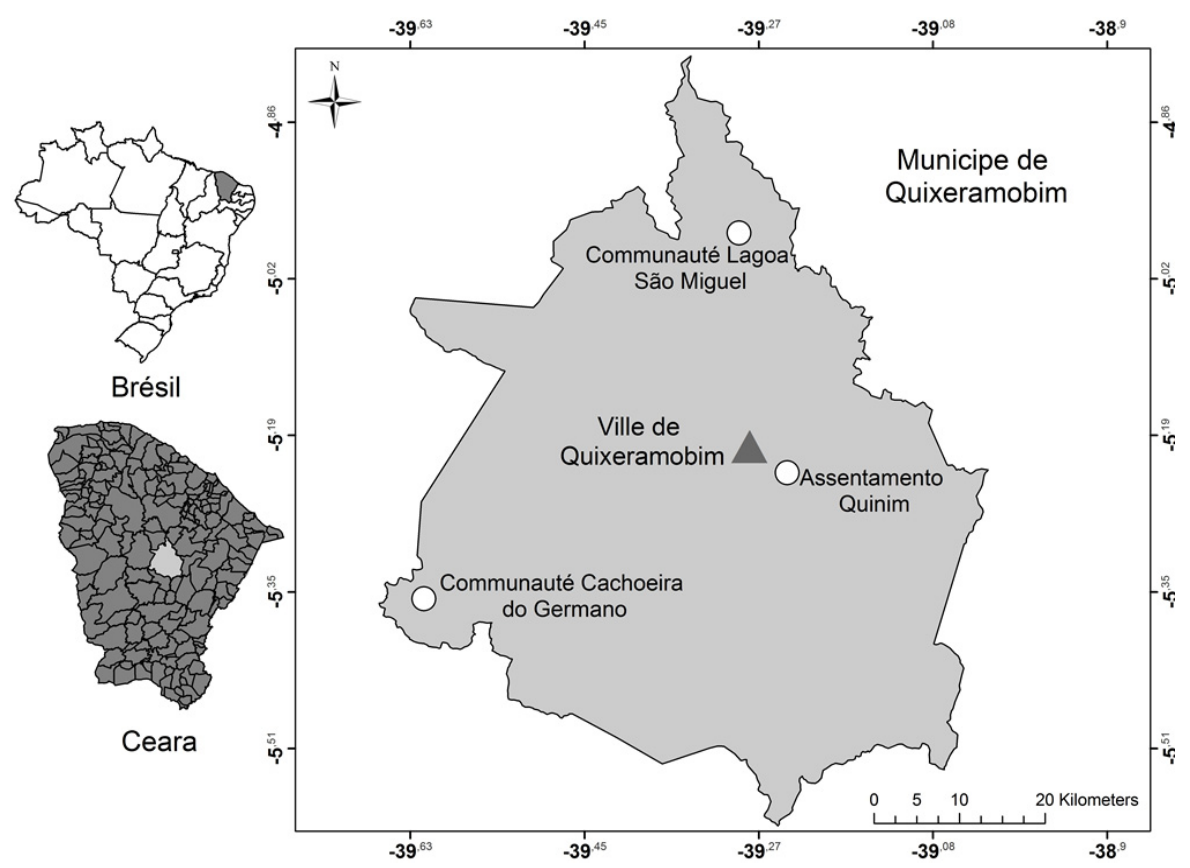

Fig. 2. Localisation des trois communautés étudiées dans le municipe de Quixeramobim - État du Ceara.

utilisée, nous analyserons les modes de gestion traditionnels de l'eau, puis celui des réseaux récemment construits, pour discuter de leur appropriation par les potentats locaux et les ruraux.

L'étude a concerné 21 foyers sur 30 à CG, 19 sur 30 à LSM et 29 sur 50 au Quinim. Ces communautés ont été choisies selon deux critères principaux: leur niveau d'isolement - facteur déterminant d'un accès plus ou moins récent et fréquent aux innovations techniques - et le mode d'occupation du territoire.

La position de CG en fait un « endroit oublié " par les porteurs de projet, comme l'exprime une habitante. Du fait de cet isolement, les habitants ont une faible expérience des transferts techniques. LSM, moins isolée que CG et plus ancrée dans des réseaux extérieurs actifs, bénéficie d'interventions techniques depuis plus de trois décennies. Le Quinim, qui est un assentamento ${ }^{5}$, c'està-dire un collectif agricole installé sur des terres publiques ou expropriées, est proche de la ville et a, de par son statut, un accès privilégié aux projets publics. Ces différentes situations nous permettent d'analyser l'impact de l'introduction d'un système technique sur les pratiques et les représentations liées aux modes de gestion des petites ressources en eau.

Le deuxième critère de différenciation de ces communautés est leur mode d'installation qui explique la densité

\footnotetext{
5 Il rassemble 50 familles qui ont obtenu des terres par le biais de la réforme agraire. Les familles habitent dans des maisons individuelles. Elles décident ensemble de la gestion des terres collectives et des règles de la vie en collectivité.
}

des liens communautaires. À CG, la communauté est composée de plusieurs groupes familiaux installés simultanément sur le territoire. À LSM, les habitants descendent en majorité du même premier occupant, et constituent ainsi une famille étendue. Au Quinim, la population est issue en majorité d'habitants qui travaillaient sur les terres de l'ancien propriétaire, les autres ont des origines diverses. L'intégration de ces liens familiaux et communautaires est nécessaire à l'analyse des dynamiques collectives.

Différents outils ont été utilisés de manière intégrée. Une approche diachronique mobilisant des récits de vie (Bertaux, 2006) a montré l'évolution des stratégies d'approvisionnement en eau. L'observation participante (Lapassade, 1991) nous a permis de saisir les attitudes du quotidien, révélatrices de logiques de gestion de la ressource. Des entretiens semi-directifs nous ont aidés à valider les observations formulées (Kaufmann, 2008).

\section{Introduction d'un réseau d'eau domestique dans un mode traditionnel de gestion de la ressource}

\section{La gestion du réseau d'eau domestique}

L'étude de la gestion des réseaux d'eau révèle de fortes similitudes à LSM et au Quinim, qui gèrent en propre l'ensemble de leur réseau. La gestion est réalisée de façon relativement autonome par les deux communautés, sur la base d'une contribution financière de 


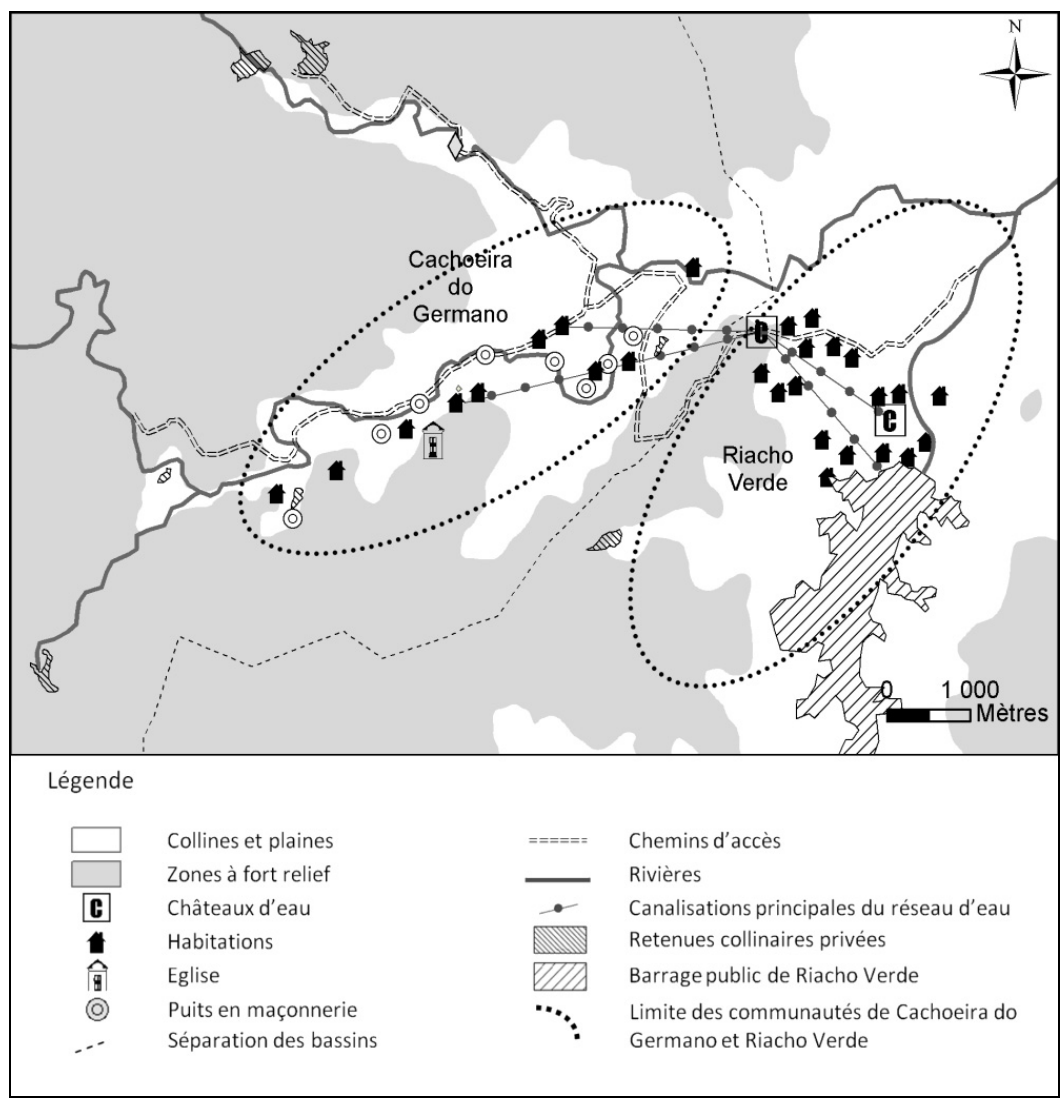

Fig. 3. Carte d'approvisionnement en eau des communautés de Cachoeira do Germano et Riacho Verde en 2010.

chaque famille liée aux usages. L'équilibre financier des réseaux dépend donc de la mesure et de la facturation des volumes consommés.

CG se différencie car elle ne possède pas son propre réseau, mais est raccordée à celui de la communauté voisine, Riacho Verde (RV). En effet, au début de l'année 2010, lors du montage du projet pour CG, l'absence de barrage sur le territoire communautaire conduit les élus locaux à choisir l'option d'un raccordement au réseau de $\mathrm{RV}$, qui possède un barrage public (Fig. 3). De ce fait, le responsable du réseau élu par les habitants de RV administre aussi l'extension qui approvisionne CG. La participation des habitants de CG à la gestion se résume au seul paiement de leur facture d'eau. La localisation des infrastructures de pompage et de stockage en dehors du territoire vécu ${ }^{6}$ explique en partie ce comportement.

On note des problèmes dans la conception initiale des réseaux : oubli des compteurs à LSM et au Quinim, capacité insuffisante du château d'eau à CG et à LSM, puissance insuffisante des moteurs au Quinim. De plus,

6 Par « territoire vécu », on définit un espace approprié par les multiples acteurs qui le composent. Cet espace influence les individus, tout comme ces derniers l'influencent en fonction des représentations et des perceptions qu'ils en ont (Brunet, 1997). le suivi technique, en théorie prévu pour assurer les capacités des habitants à gérer et maintenir en fonctionnement l'infrastructure, est inexistant dans les trois communautés. Les difficultés de trésorerie pour l'achat de biens ou de matériel d'entretien courant du réseau (compteurs, canalisations, pompes) sont récurrentes. Le mode de gestion ne permet pas d'épargner assez d'argent pour l'entretien.

Les problèmes de conception initiaux et l'absence d'accompagnement des populations locales poussent les responsables du réseau à solliciter un acteur extérieur (technicien, élu local) pour accéder à de nouvelles pièces mécaniques. La gestion et la manutention des réseaux reposent donc largement sur des arrangements entre les populations locales et des acteurs externes aux communautés, alors que, selon les porteurs du modèle d'action publique, les procédures, pourtant définies uniquement au niveau de l'État et des bailleurs de fond, doivent conduire les communautés à prendre en charge ces responsabilités.

Afin de discuter les opportunités offertes aux populations locales de modifier leurs rapports de dépendance avec l'extérieur, l'analyse est centrée sur les transformations apportées par les réseaux d'adduction en eau sur ces relations. 


\section{Les relations de dépendance dans les modes de gestion traditionnels des petites ressources en eau}

Par « traditionnel », on désigne des éléments du passé qui sont transmis de génération en génération selon un processus dynamique à l'opposé d'une réalité figée dans le temps (Balandier, 1967).

Les stratégies traditionnelles d'approvisionnement en eau observées dans les trois communautés sont typiques de la région (Sabourin et al., 2002). La formulation des règles permettait $d^{\prime}$ assurer une gestion des ressources qui visait d'abord l'approvisionnement humain et animal, vital dans un milieu marqué par le manque d'eau.

L'accès à l'eau et son partage entretenaient des liens de solidarité à l'intérieur des communautés. À CG et à LSM, la gestion de l'eau était au quotidien réalisée à l'échelle du noyau familial, surtout pour l'approvisionnement en eau de boisson. L'usage de l'eau des citernes pouvait s'étendre aux voisins et aux amis de la famille, et l'accès aux puits était permis à ceux qui avaient participé à leur construction. Ainsi, l'eau était un objet d'intégration des habitants. La solidarité concernait toute la famille étendue, voire la communauté en cas de pénurie. À CG, l'installation des foyers le long du même axe d'écoulement a créé un trait d'union, renforcé par le chemin qui suit aussi cet axe.

$\mathrm{Au}$ quotidien, et surtout lors des pénuries, les habitants étaient aussi plus ou moins dépendants des «faveurs" des fazendeiros pour leurs besoins en eau domestiques et pastoraux. Ces relations clientélistes sont souvent associées à des relations d'exploitation (Lanna, 1995), alors que des valeurs humaines lui sont aussi rattachées (Sabourin, 2007). À LSM, le principal propriétaire terrien de la communauté est également le chef de la famille étendue. Les habitants ont longtemps interagi autour du premier réservoir construit par celui-ci. Il est appelé «padrinho» (parrain), celui qui aide, par les personnes âgées. À CG et à LSM, les grands propriétaires terriens sont nommés « vizinhos » (voisins, de la communauté) ou "compadres » (compères), ceux sur qui l'on peut compter. Ces dénominations font référence à une proximité géographique et à des liens d'interconnaissance. Au Quinim, un premier réseau d'eau existait déjà à l'époque de la fazenda. L'ensemble des usagers dépendaient alors d'un système technique contrôlé par le fazendeiro. L'eau était un objet d'interaction selon une obligation morale du patron envers ses travailleurs, comprise dans une relation de devoirs mutuels.

Un autre élément important des stratégies d'approvisionnement en eau domestique était la pénibilité des tâches d'approvisionnement traditionnel, particulièrement à CG et LSM. Les distances entre les maisons et les points d'eau sont importantes, entre 3 et $6 \mathrm{~km}$. L'épuisement des petites ressources en eau locales obligeait fréquemment les populations à utiliser des ressources en eau privées, distantes et situées en-dehors du territoire de la communauté. Le lavage du linge était aussi une tâche difficile, car les femmes devaient puiser l'eau manuellement dans des puits et rapporter les bassines jusqu'à leur foyer : " Ma tête ne supporte plus de transporter tant d'eau jusqu'ici [sa maison]. » Elles décrivent ces tâches en termes de "souffrances " (mains abîmées, douleurs aux jambes et au dos) qui « donnaient parfois envie de pleurer $»$.

La dépendance des populations locales, vis-à-vis de l'extérieur, variait selon les liens communautaires, les saisons (des pluies et sèches) et les événements de sécheresse. La mise en œuvre du projet communautaire d'adduction d'eau a-t-elle modifié ce paysage social et mental?

\section{Déplacement des relations de dépendance}

L'analyse de l'introduction du réseau d'eau domestique nous amène à deux principaux constats, communs aux trois communautés, et illustrés ci-dessous : le changement de la nature du clientélisme et la persistance d'une imposition descendante des décisions.

En théorie, l'association doit formuler la demande d'accès à un projet, celle-ci est ensuite évaluée par le conseil municipal de développement durable puis transmise au secrétariat du Développement agraire de l'État, qui doit l'approuver. Les acteurs du municipe sont logiquement exclus des procédures d'attribution, afin de réduire leurs possibilités de détourner l'argent des projets.

On observe en réalité une confusion des procédures d'accès aux projets, induite par une absence de critères clairs et rigoureux pour l'octroi des infrastructures d'adduction d'eau par l'État. Les associations communautaires, qui n'ont pas de réel poids décisionnel, doivent solliciter les notables locaux du municipe pour espérer un accès à des financements. Du fait de cette procédure, les habitants de CG adoptent un comportement fataliste face à ce rapport de dépendance.

Le cas de CG montre bien la nécessité de l'intervention d'un conseiller municipal, à plusieurs niveaux, notamment pour convaincre les habitants de RV qui refusaient l'extension de leur réseau. Ils défendaient en effet l'exclusivité de leur accès au barrage public, qu'ils s'étaient appropriés (Burte et al., 2009), car ils craignaient que le branchement de CG sur leur réseau ne fasse baisser le niveau du réservoir.

L'ensemble des habitants de CG ne remettent pas en cause l'intervention de l'élu local. Ils associent l'installation du réseau à la période électorale et l'interprètent comme une intervention politique. Le président de l'association communautaire de CG nomme l'élu local «representante », il le désigne en tant que « représentant 
politique de la communauté ». Cela illustre un déplacement de la nature du clientélisme. Traditionnellement, les ruraux dépendaient des fazendeiros pour leur survie et l'accès au travail et aux soins (Caron et Sabourin, 2001). Actuellement, les ruraux dépendent des élus locaux pour accéder aux soins, mais surtout pour bénéficier des projets et assurer leur visibilité auprès des pouvoirs publics.

La proximité entre habitants et potentats locaux a aussi changé. Les fazendeiros appartenaient au monde rural en habitant les campagnes. Les élus sont des acteurs urbains qui interviennent essentiellement pour des enjeux électoraux et de captation de votes. Cette distanciation s'observe dans les trois communautés.

À l'instar des relations de dépendance entre les habitants et les fazendeiros, la reproduction de la relation clientéliste avec les élus locaux comporte des valeurs humaines. Les ruraux expriment leur respect envers les élus locaux, qui cherchent, quant à eux, une légitimité et une reconnaissance de leur statut.

La confusion des procédures d'accès aux projets maintient aussi une logique descendante de l'introduction des projets. À CG, la décision d'installation du réseau provient de l'élu local : «Ils [les élus locaux] ont inventé ça [l'installation du réseau]. » Pourtant, le président de l'association argumente sa réelle participation à la décision: "J'ai pensé à ça [la proposition du conseiller municipal d'installer un réseau] et j'ai choisi de canaliser l'eau parce que l'eau c'est la vie. Ça permet de se nourrir si besoin ; si on a soif, ça permet de boire. » Le rapport de proximité entre les deux hommes a facilité l'instrumentalisation de la symbolique de l'eau par l'élu local.

La logique descendante dans les procédures d'introduction du réseau d'eau se retrouve à LSM et au Quinim : la proposition de construire un réseau (LSM) ou de rénover l'ancien (Quinim) vient des techniciens de l'organisme d'État chargé de la construction des infrastructures: la Superintendance des ouvrages hydrauliques (Sohidra).

Enfin, il faut noter l'apparition d'une nouvelle relation de dépendance de la communauté de CG, envers celle de RV. Les habitants de CG dépendent de la pompe, de l'açude, du château d'eau et du gestionnaire de RV. Mais les deux parties du réseau ne sont pas gérées dans les mêmes conditions de facturation, ce qui induit la suspicion. Au lieu de créer de nouvelles relations intercommunautaires et une gestion concertée de l'eau, le projet favorise l'opposition de la population de CG et de $\mathrm{RV}$. La conception sociale (absence de discussion sur les modalités d'usages du réseau, précipitation de l'élu local pour des raisons électorales) et technique (oubli des compteurs d'eau) du projet a éloigné les communautés au lieu de les rapprocher.

Un projet présenté comme participatif et émancipateur par les porteurs du modèle d'action publique ne se traduit pas ainsi dans la réalité, du fait de l'ambiguïté des procédures, de l'absence de concertation entre communautés et en leur sein, et d'une mise en œuvre inachevée due à des financements limités et à l'absence de planification.

\section{Dépendance croissante des populations rurales envers le réseau d'eau}

Les habitants expliquent que la majorité des personnes ont des alternatives au réseau pour se procurer l'eau de boisson. C'est pourquoi l'eau du système n'est pas traitée. En réalité, l'eau de pluie stockée dans les citernes, plus douce que celle délivrée par le réseau, est privilégiée. Cette eau est aussi consommée pour des usages domestiques. Cette appropriation est renforcée par les pannes récurrentes des réseaux, pendant lesquelles les habitants ne vont plus chercher l'eau loin de leurs foyers, mais préfèrent l'eau des citernes, dont le stock est vite consommé, rendant alors vital le recours aux camions-citernes... qui dépend de l'intervention d'un élu local. Ainsi, l'introduction du réseau n'élimine pas la nécessité d'une intervention chargée symboliquement par l'héritage clientéliste.

L'absence de traitement de l'eau du réseau peut aussi être interprétée comme la conséquence d'une appropriation des usages de l'eau du réseau par les habitants. À l'origine destiné à la consommation humaine, le réseau est aujourd'hui utilisé par la plupart des habitants pour des usages domestiques variés (toilette familiale, sanitaires, lessive, nettoyage de la maison), pour l'abreuvement des animaux et, souvent, pour l'arrosage d'un petit jardin. En effet, le réseau lève la contrainte des distances que le système traditionnel imposait aux tâches domestiques, désormais réalisées dans les arrière-cours. Dans les trois communautés, l'innovation technique est vécue comme l'élément qui a le plus amélioré le quotidien, après l'électrification (Photo). Le système technique " réseau d'eau » donne aux populations le sentiment que le risque de manque d'eau n'existe plus ; ainsi des familles à faibles revenus font-elles des investissements lourds, tels que l'achat de machines à laver. La facilité d'accès à l'eau induit ces comportements, rendus possibles grâce aux allocations familiales et aux crédits. Le nouvel accès à l'eau favorise aussi des pratiques de jardinage et d'élevage qui permettent une réduction des dépenses des ménages.

Ces comportements témoignent d'un sentiment de confiance presque excessif envers le système technique et d'une forte dépendance des habitants envers le réseau. Or, celui-ci ne sécurise pas la ressource elle-même. Une responsable des projets São José explique qu'aucune étude sur la sécurité des barrages n'a été réalisée jusqu'alors. Dans le cas d'une sécheresse pluriannuelle, les açudes de RV, de LSM et du Quinim peuvent s'assécher 


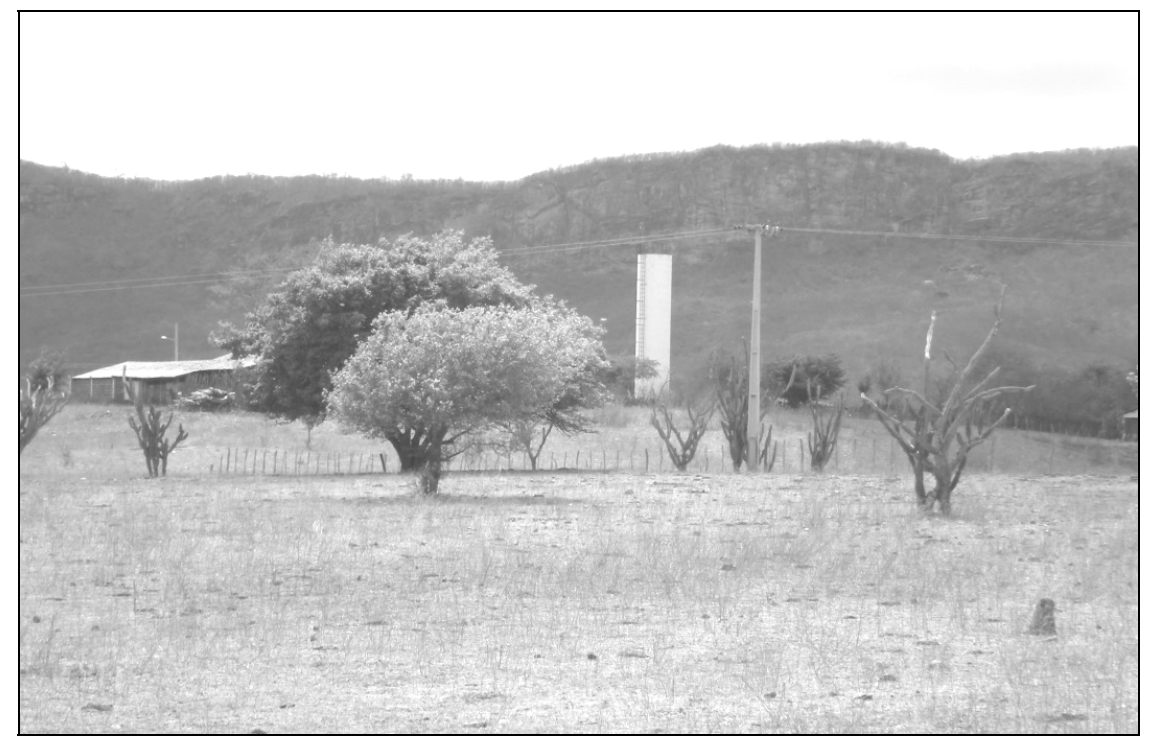

Photo. Un paysage de Lagoa São Miguel (Source : Collard, 2010).

Le château d'eau est un élément central dans le paysage semi-aride, au même titre que le poteau et les câbles du réseau électrique.

(Burte et al., 2009). Même si la population n'ignore pas ce risque, la mémoire des grandes sécheresses est courte. Les habitants ont du mal à imaginer qu'un tel événement puisse se reproduire, puisque l'eau arrive maintenant jusque chez eux. Leur méfiance porte sur la fiabilité technique du réseau victime de coupures d'eau prolongées, mais pas sur les capacités de l'açude.

Bien que la nature du clientélisme ait changé, les anciennes figures locales n'ont pas disparu du paysage social du monde rural. Les grands propriétaires terriens continuent de contrôler l'accès et la distribution de l'eau des cours d'eau et des barrages publics situés sur leurs terres. Ainsi, en cas de sécheresse pluriannuelle, le risque sur l'approvisionnement en eau domestique et sur le bétail n'étant pas réduit par le réseau, le recours aux fazendeiros restera incontournable ; leurs « faveurs » représentent donc toujours une garantie pour les populations rurales d'atténuer les risques.

\section{Conclusion}

En termes d'appropriation, la réalisation d'un réseau d'eau représente, dans les trois cas étudiés, un transfert technologique sans véritable innovation sociale, même $\mathrm{si}$, à l'origine, pour les porteurs du modèle d'action publique et les bailleurs de fonds, le fait d'en faire un objet communautaire, porté par une association, se voulait une rupture. Il reste en effet empreint de l'héritage sociohistorique : d'une part, parce que les procédures d'obtention des projets, de mise en œuvre et d'entretien sont ancrées dans des jeux politiques clientélistes, et d'autre part, parce que la logique d'action publique descendante persiste.
Le discours politique sur les projets d'adduction d'eau révèle une vision technocratique traditionnelle du développement (Dupré, 1991). Les sociétés rurales sont perçues comme des systèmes fermés, pour lesquels l'intervention extérieure est pensée selon un objectif prédéfini qui ne prend pas en compte, ni ne valorise, les connaissances et les représentations locales.

On voit qu'ici l'action publique n'a pas entraîné une réelle action collective. Il est important de ne pas généraliser ce propos qui repose sur l'analyse de trois cas locaux $\mathrm{du}$ Nordeste. D'autres études ont montré l'existence d'actions collectives paysannes dans cette région $\mathrm{du}$ Brésil (Sabourin et al., 2004 ; 2005) pour la construction de citernes, l'organisation de banques de semences communautaires ou la gestion collective des forêts sèches. Néanmoins, les conditions d'articulation entre action collective et action publique sont différentes de celles analysées dans cet article. En effet, l'action publique appuyait et reconnaissait des dispositifs collectifs préexistants à l'intervention. Certains de ces projets étaient accompagnés d'un partenariat étroit entre agriculteurs, techniciens et chercheurs. Enfin, les interventions répondaient à une demande locale. Ainsi, le contexte d'intervention est différent de celui dans lequel s'inscrit l'introduction des projets de réseau d'adduction d'eau domestique, qui reste dominée par une initiative extérieure.

L'introduction de médiateurs - qui existent ailleurs dans le Nordeste, comme les syndicats ou les techniciens, mais qui ne sont pas actifs dans notre cas d'étude - et la circulation d'informations auraient été pertinentes pour aider à la coordination des actions, et donner aux populations locales, non seulement des compétences, mais aussi des opportunités pour s'adapter aux nouvelles 
situations (De Munck, 2008 ; Tonneau et al., 2009). Mais, il n'y a pas eu d'apprentissage des techniques ni de formation de leaders communautaires, comme prévu dans le projet initial.

Dans un contexte où l'oligarchie agraire constitue historiquement un contre-pouvoir, structurant des inégalités sociales, l'introduction d'un système technique visant à répondre aux besoins des populations « oubliées " n'est pas neutre. La volonté affichée des gouvernements, tant au niveau des États qu'au niveau fédéral, de favoriser l'autonomie des populations locales par un transfert de gestion qui déconstruise l'objet « eau » en tant qu'instrument de pouvoir, pour en faire un objet de participation locale, ne se retrouve pas dans les faits. L'absence de concertation dans la gestion de l'eau persiste, ce qui ne permet pas de renverser la dynamique de décision descendante.

En réalité, l'intervention publique a créé une nouvelle forme de dépendance de la population locale, dans laquelle les enjeux électoraux mêlés à l'attribution des marchés publics sont des facteurs structurants. Ces projets de petites infrastructures, dont la population locale est demandeuse, sont des objets instrumentalisés par les pouvoirs locaux. Les périodes électorales sont propices à leur multiplication. On assiste ainsi à une fuite en avant dans les dépenses, similaire à celle souvent observée pour les ouvrages de grande hydraulique, du fait de l'absence de concertation dans la planification et de l'accumulation d'investissements peu efficaces.

Le système technique n'a donc pas généré de réelle autonomisation des populations locales. Les affects et les représentations des individus n'ont pas été mis en relation avec les cadres sociaux de la région (Darré, 1999) pour que le système technique soit discuté et élaboré avec la population locale, et non pas uniquement avec la sphère politique ou technique.

L'analyse de notre cas d'étude nous permet de participer aux discussions sur les perceptions des cadres sociaux, que sont l'héritage sociohistorique clientéliste et les relations de subordination des populations locales reproduites par l'oligarchie locale, souvent considérés comme figés par les bailleurs de fonds internationaux, les porteurs du modèle d'action publique et les techniciens. En réalité, la nature du clientélisme a changé. Plutôt que de nier son existence dans le paysage social, il serait plus réaliste de le prendre en compte, avec ses évolutions, dans l'élaboration des projets.

Il nous a semblé important de mettre l'accent sur les conséquences à long terme d'un système technique pensé en dehors de ses usagers, sans pour autant négliger le fait que l'introduction d'un réseau d'eau soit une avancée intéressante pour la population locale. Le réseau améliore, en effet, le quotidien des personnes en réduisant la pénibilité des tâches domestiques et en permettant l'émergence de petits élevages et de jardins à proximité des habitations.
Pour aller plus loin dans l'analyse du déplacement de la nature du clientélisme, il serait aussi intéressant de traiter des conséquences d'un approvisionnement individuel en eau, sur les pratiques collectives d'approvisionnement ou de préservation des ressources. Le réseau induit une distanciation entre ressources et usages, chacun ayant son robinet à domicile. Nous avons ainsi observé des comportements opportunistes qui « peuvent induire certains acteurs à ne pas participer à des actions collectives »(Ostrom, 1992). Il ne s'agit pas d'adhérer à un «populisme de l'action» (Olivier de Sardan, 1990). L'altruisme paysan n'est pas une donnée sociale. Il existe par nécessité. Cependant, l'action collective produit du lien social, nécessaire pour penser la gestion des petites ressources locales. Sabourin (2007) propose de s'intéresser « aux relations et aux structures de réciprocité [...] là où elles subsistent », pour créer du capital social. Comme cela a déjà été observé dans d'autres communautés de cette région (Burte et al., 2009), les pratiques traditionnelles et les mécanismes de réciprocité ont tendance à disparaître et les tensions amont/aval à se renforcer. Cela devrait conduire à mettre en place de nouvelles formes de gestion de l'eau, à l'échelle du « territoire de l'eau » pour dépasser le caractère très local et purement technique de la gestion actuelle.

\section{Remerciements}

Ces travaux ont bénéficié de l'appui de la Funcap (Fondation cearence d'appui à la recherche), de la Funceme (Fondation du Ceará de météorologie et ressources hydriques), du Cirad-UMR G-Eau de Montpellier, de 1'USP (Université de São Paulo), de l'UFC (Université fédérale du Ceará) et d'AgroParisTech.

\section{Références}

Abramovay, R., 2002. Crédit rural et politiques publiques dans le sertão brésilien, Tiers-Monde, 172, 761-782 .

Akrich, M., 1989. La construction d'un système sociotechnique, Anthropologie et Sociétés, 13, 2, 31-54.

Amman, S.B., 1985. Ideologia do desenvolvimento de comunidades no Brasil, São Paulo, Cortez.

Andrade, C.M., 1986. A intervenção do estado e a seca no Nordeste do Brasil, Revista de Economia Politica, 6, 4, 125-130.

Araujo, J.C., Molinas, P.A., Joca, E.L.L., Barbosa, C.P., Bemfeito, C.J.D.S., Belo, P.S.D.C., 2005. Custo de disponibilização e distribuição da água por diversas fontes no cearà, Revista Econômica do Nordeste, 36, 2, 281-307.

Balandier, G., 1967. Anthropologie politique, Paris, PUF.

Bertaux, D., 2006. L'enquête et ses méthodes : Le récit de vie, Paris, Armand Colin.

Bleil, S., 2003. Tensions entre le communautaire et le public: l'expérience d'un assentamento des "sans terre », in Carrel, M., Guerrero, J.-C., Barri, C., Marquez, A. (Eds), Le Public en action. Usages et limites en sciences sociales, Paris, L'Harmattan, 179-198. 
Brunet, R., 1997. Champs et contrechamps : raisons de géographe, Paris, Belin, coll. Mappemonde.

Burte, J., Jamin, J.-Y., Coudrain, A., Frischkorn, H., Martins, E.S., 2009. Simulations of multipurpose water availability in a semi-arid catchment under different management strategies, Agricultural Water Management, 96, 8, 1181-1190.

Caron, P.,Sabourin, É., 2001. Paysans du sertão : mutations des agricultures familiales dans le Nordeste du Brésil, Montpellier, Quae.

Cohen, M., Duqué, G., 1989. Sécheresse et modèle de développement. Le cas du projet Sertanejo, in Bret, B. (Ed.), Les Hommes face aux sécheresses. Nordeste brésilien, Sahel africain, Paris, IHEAL, 385-389.

Darré, J.-P., 1999. La production de connaissance pour l'action: Arguments contre le racisme de l'intelligence, Paris, MSH-Inra.

De Castro, J., 1946. Geografia da fome : a fome no Brasil, Rio de Janeiro, O Cruzeiro.

De Munck, J., 2008. Qu'est-ce qu'une capacité ?, in De Munck, J., Zimmerman, B. (Eds), La liberté au prisme des capacités, Paris, EHESS, 21-49.

Dupré, G., 1991. Introduction, in Dupré, G. (Ed.), Savoirs paysans et développement, Paris, Orstom, Karthala, 17-31.

Duqué, G., 2008. "Conviver com a seca » : contribuição da Articulação do Semi-Árido/ASA para o desenvolvimento sustentável, Desenvolvimento e Meio Ambiente, 17, 133-140.

Durousset, É., 2001. À qui profitent les actions de développement? : la parole confisquée des petits paysans (Nordeste, Brésil), Paris, L'Harmattan.

Durousset, É., Duqué, G., 1998. As relações entre pequenos produtores e poderes públicos : o caso de um projeto de irrigação no semi-árido, Raizes, 18, 79-89.

Garfinkel, H., 2007. Recherches en ethnométhodologie, Paris, PUF (1967 pour l'édition originale).

Geffray, C., 1996. Le modèle de l'exploitation paternaliste, Lusotopie, 153-159.

Harmonicop, 2005. Apprendre ensemble pour gérer ensemble: améliorer la participation à la gestion de l'eau., Université d'Osnabrück.

Jacobi, P.R., Tristão, M., Gonçalves Correa Franco, M.I., 2009. A função social da educação ambiental nas praticas colaborativas : participação e engajamento, Cadernos Cedes, $29,77,63-79$.

Jamin, J.-Y., Bouarfa, S., Poussin, J.-C., Garin, P., 2011. Les agricultures irriguées face à de nouveaux défis, Cahiers Agricultures, 20, 1-2, 10-15.

Kaufmann, J.-C., 2008. L'Entretien compréhensif, Paris, Armand Colin.

Kuper, M., 2011. Des destins croisés : regard sur 30 ans de recherches en grande hydraulique, Cahiers Agricultures, 20, 1-2, 16-23.

Kuper, M., Errahj, M., Faysse, N., Caron, P., Djebbara, M., Kemmoun, H., 2009. Autonomie et dépendance des irrigants en grande hydraulique : observations de l'action organisée au Maroc et en Algérie, Natures Sciences Sociétés, 17, 248-256.

Lanna, M.P.D., 1995. A divida divina: troca e patronagem no Nordeste brasileiro, Campinas, Unicamp.
Lapassade, G., 1991. L'Ethnosociologie, Paris, Méridiens Klincksieck.

Latour, B., 1991. Nous n'avons jamais été modernes, Paris, La Découverte.

Mainguet, M., 2003. Les sécheresses et le génie créateur de l'homme dans les milieux secs: nouvelle géographie de l'adaptation?, in Mainguet, M. (Ed.), L'Homme et la sécheresse, Paris, Masson.

Marzouk, Y., 1991. Histoire des conceptions hydrauliques étatiques et paysannes en Basse Casamance, Sénégal, 19601990, in Dupré, G. (Ed.), Savoirs paysans et développement, Orstom, Karthala, 61-97.

Molle, F., 1994. Politique de l'eau, irrigation et société : le cas du Nordeste brésilien, Les Cahiers de la Recherche Développement, 37, 19-32.

Moscovici, S., 1961. La Psychanalyse, son image et son public, Paris, PUF.

Olivier de Sardan, J.-P., 1990. Populisme développementiste et populisme en sciences sociales : idéologie, action, connaissance, Cahiers d'Études Africaines, 30, 120, 475-492.

Ostrom, E., 1992. Crafting Institutions for Self-Governing Irrigation Systems, San Francisco, Institute for Contemporary Studies Press.

Pinton, F., 2009. De la paysannerie française aux peuples de la forêt amazonienne, Études Rurales, 183, 201-218.

Sabourin, É., 2007. L'entraide rurale, entre échange et réciprocité, Revue du Mauss, 30, 198-217.

Sabourin, É., Duqué, G., Diniz, P.C.O., Do Socorro de Lima Oliveira M., Granchamp Florentino, L., 2005. Reconnaissance publique des acteurs collectifs de l'agriculture familiale au Nordeste, Cahiers Agricultures, 14, 1, 111-115.

Sabourin, É., Silveira, L., Sidersky, P., 2004. Production d'innovation en partenariat et agriculteurs expérimentateurs au Nordeste du Brésil, Cahiers Agricultures, 13, 2, 203-210.

Sabourin, É., Sidersky, P., Matos, L.C., Trier, R., 2002. Gestion technique vs gestion sociale de l'eau dans les systèmes d'agriculture familiale du Sertão brésilien, Sécheresse, 13, 4, 274-283.

Soares, R.P., 1997. Avaliação do financiamento do banco mundial ao programa de apoio ao pequeno produtor rural do Nordeste (PAPP), Planejamento e Políticas Publicas, 15.

Tonneau, J.-P., Piraux, M., Coudel, É., (de) Azevedo, S.G., 2009. Évaluation du développement territorial comme processus d'innovation et d'institutionnalisation : le cas du Territoire du Alto Sertão do Piauí e Pernambuco au Nordeste du Brésil, VertigO, 9, 3, 1-14.

Tonneau, J.-P., Sidersky, P., Eloy, L., Sabourin, É., 2010. Dynamiques et enjeux des agricultures familiales au Brésil, dossier « Le Brésil, ferme du monde? », Géoconfluences.

Venot, J.-P., Cecchi, P., 2011. Valeurs d'usage ou performances techniques : comment apprécier le rôle des petits barrages en Afrique subsaharienne?, Cahiers Agricultures, 20, 112117.

Vinck, D., 1999. Les objets intermédiaires dans les réseaux de coopération scientifique : contribution à la prise en compte des objets dans les dynamiques sociales, Revue Française de Sociologie, 40, 2, 385-414. 